\title{
Getting rid of the blues
}

\author{
LEONARD BROSGOLE \\ St John's University, Jamaica, New York
}

\begin{abstract}
Cataract removal from the left eye resulted in color distortion in that eye; the entire visual world seemed to be bathed in blue light. Reasoning that this may have been due to a conditioned color aftereffect, a perceptual adaptation procedure was devised to correct the problem. Normal color vision was restored in the eye after six 10-min adaptation sessions.
\end{abstract}

This paper describes a procedure that I devised to extinguish a presumed conditioned aftereffect that appeared following the removal of a nuclear cataract. I was both patient and subject, and the procedure described here was developed in a home environment under conditions of controlled panic. Therefore, the procedure was not subjected to rigorous control, but is suggested for future laboratory research. The distressing aftereffect of surgery was that the entire world appeared to be bathed in various shades of blue; the problem was how to get rid of the blues.

I have been severely myopic since early childhood. The cataract was in the left eye, which had a visual acuity of $20 / 400$, with correction, which was measured prior to the cataract becoming so dense as to preclude making any meaningful measurements. The cataract had been in the eye for a minimum of 17 years prior to removal. I resisted surgery for fear of retinal detachment, but finally consented when I was advised that the cataract was dangerously close to bursting.

The first hint of a problem was a slight blurring of vision. I am not sure how long this persisted, but this was followed by a sudden marked discoloration of the visual field. It was as if I were viewing the world through an orange-gold (and at times brownish) film. This discoloration seemed to occur almost overnight about 17 years before surgery and grew more dense as the years progressed. Ten years prior to surgery, I had lost almost all articulated vision. Oddly enough, however, color discrimination remained almost intact; the only problems were with yellows, which looked orange-gold, and blues, which had a grayish cast. There was no distinction between the left and right eyes in judging and matching colors. Just prior to surgery, it was as if I were looking through a translucent, three-dimensional chip of crystal filled with brownish-black ridges. The visual world was broken apart, as though the lens were multifaceted. In addition, there were black spots, or holes, in the visual field. There was no problem of glare under normal levels of illumi-

Address correspondence to L. Brosgole, Department of Psychology, St. John's University, Grand Central and Utopia Parkways, Jamaica, NY 11439. nation. However, very bright light tended to diffuse, creating the impression of looking into a ganzfeld.

The surgery was quite successful, in that there was no retinal detachment and acuity improved to 20/200 with subsequent correction. When sight in the eye began to return about $3 \mathrm{~h}$ after surgery, however, I noticed that everything seemed to be cast in a sky-blue light. This did not concern me at first, as I attributed this to a side effect of surgery. But the blue persisted over the days to follow; all visible space was filled with blue. The color would lighten into a pleasing blue on sunny days and darken into a deep purple at dusk, or when the skies were filled with black storm clouds. In addition to the voluminous blue, there was also some disturbance in properly sensing some surface colors. Whites looked powder blue and yellows looked green. Reds appeared off-color and washed out, and pinks were achromatic. My own skin appeared to be colored a deoxygenated, cadaver blue. For whatever reason, the absence of red did not disturb me, but the presence of blue was quite distressing.

I visited the surgeon for a second time 8 days after surgery and, at that point, shared my problem with him. I expressed my fear that there might be some type of retinal pathology, but also speculated that I might have been the victim of a conditioned color aftereffect. He reassured me that the problem was not retinal, but most likely was determined centrally. He indicated that my problem was not uncommon (hence this paper), and that it could last anywhere from several weeks to a lifetime. He could not provide any demographics related to the problem and was unable to define the disturbance so that I could track down the phenomenon in the literature. As a result of our discussion, I was convinced that I was experiencing a conditioned color aftereffect of the type described by Kohler (1962). I reasoned that if this were the case, then it should be possible to design a counterconditioning procedure to restore normal color vision. Thus, I directed myself at getting rid of the blues.

The logic of the procedure used was to neutralize the blue aftereffect by creating a counteracting yellow aftereffect, under a condition of reafferent stimulation. To this end, I added blue to the field and, with my left eye, watched my hand as it was moved from side to side voluntarily within the field. 


\section{METHOD}

Subject

The author was both subject and observer. At the date of surgery he was 55 years old. He had had a nuclear cataract in the left eye for a minimum of 17 years. Because of congenital myopia and nystagmus, the vision in that eye had been exceptionally poor since childhood. As a result, there was a tendency to suppress that eye and the subject was never able to fuse binocularly. The subject was Caucasian, right-eye dominant, and left-handed.

\section{Materials}

As previously noted, the procedure was run in the home. Therefore, fixtures within and around the home were used to create an adaptation field and test stimuli. A blue surround was created by placing my face as close as possible to a medium-blue bathroom sink. This blue field subtended a visual angle of approximately $120^{\circ}$ laterally. I was unable to get any closer to the surface of the sink, as I would have impaled the bridge of my nose upon its single-lever faucet. The stimuli used to test for perceptual adaptation were a yellow sodium street lamp, an outdoor 60 -W bug light, the deep gold torch-like flame of a Zippo pipe lighter, a 100-W soft-white incandescent bulb in an outdoor fixture with a white frosted globe, and a white enamel refrigerator door. Thus, three yellow and two white stimuli were used to test for perceptual adaptation.

\section{Procedure}

The procedure, run after dark for 6 consecutive nights, consisted of placing my face within the sink and turning my head slightly to the right, so that the center of the sink was directly in front of my left eye. My left hand was wedged into the sink and the dorsum of my hand, wrist, and part of my forearm were in view. With my right eye closed, I tried to fixate the center of the field as I moved my left arm back and forth along an arc from southeast to northwest. Each of the six sessions lasted approximately $10 \mathrm{~min}$, after which time I assessed the colors of the aforementioned test stimuli.

\section{RESULTS AND DISCUSSION}

Theoretically, any reafferent movement (e.g., snapping the fingers or tapping a foot) should have been sufficient to produce adaptation. However, my hand was placed and moved within the sink for a pragmatic reason-to occlude the sight of the chromium drain. As it turned out, viewing the hand provided critical information regarding the onset and subsequent completion of the adaptation process.

As mentioned earlier, the surface of the skin appeared to be blue and lifeless. About $7 \mathrm{~min}$ into the first session, little striations of pink seemed to emanate from the hand and forearm. These pink tinges floated above the surface of the skin and would flash in and fade out. I continued the procedure for approximately $3 \mathrm{~min}$ longer, but the experience did not change. That is, the quality of pink was not enduring and it did not adhere to the surface of the skin. When I subsequently viewed the test stimuli, I noticed that whites appeared ever so slightly less blue, and yellow was just noticeably less green.
In the second session, the skin became more lifelike, as threads of surface pink mixed with the blue. The white test stimuli became even less blue, and the yellows appeared to be a very pale green. Skin tone started to normalize in the third session. The color of the limb appeared to be uniform, but was very desaturated. The white test stimuli retained a slight blue cast. The yellow stimuli appeared to be white in the center; this white bead, or nucleus, was surrounded by pale green.

Skin tone progressively darkened and blue faded from the white test stimuli during the next two sessions. With regard to the yellow test stimuli, the white center core appeared to be surrounded by a desaturated yellow after the fourth session. By the end of the fifth session, all of the yellow stimuli appeared to be a uniform pale yellow. The visual world completely normalized after the sixth session; the blue had disappeared on the morning of the seventh day and the procedure was discontinued.

It is interesting that $I$ began to notice a disturbance in color vision in the right eye as normal color vision was being restored in the left. Although a cataract was detected in the right eye 4 years ago, it did not seem to give rise to any difficulty in color recognition. However, I have become aware that blues contain some gray, whites are off-white toward yellow, and yellows tend to appear gold when seen through the right eye. This impairment has become worse over the months following surgery on the left eye. On the bright side, however, there may be a second study in the making; only next time, the data will be collected systematically.

It is fully recognized that the changes in color vision attributed to an adaptation procedure may have occurred spontaneously over time. However, I do not believe this to be the case, because each adaptation session was followed immediately by changes in the mode of appearance of the various test stimuli. Nonetheless, it would be important to run other subjects through an adaptation procedure under controlled conditions. Unfortunately, my university does not have a hospital affiliation and we do not have access to cataract patients. Therefore, this study, conducted with a bathroom sink and a bug light, should be viewed as a preliminary piece of research that is intended to encourage others to explore methods for correcting color disturbances produced by cataract removal.

\section{REFERENCE}

KoHLER, I. (1962). Experiments with goggles. Scientific American, 206, 62-72.

(Manuscript received May 3, 1993.) 